\title{
Prevalence of morbidity and mortality of diabetes mellitus in a rural community cohort
}

\author{
M Abu Sayeed ${ }^{1^{*}}$, Parvin Akter Khanam ${ }^{2}$, Akhter Banu ${ }^{3}$, Khandaker Abul Ahsan ${ }^{4}$, Fazlul Haq ${ }^{4}$ \\ ${ }^{1}$ Department of Community Medicine, Ibrahim Medical College, Segunbagicha, Dhaka, Bangladesh; \\ ${ }^{2}$ Epidemiology, Research Division, Bangladesh Institute of Research and Rehabilitation in Diabetes, \\ Endocrine and Metabolic Disorders (BIRDEM), Dhaka; ${ }^{3}$ Institute of Nutrition and Food Science, \\ University of Dhaka; ${ }^{4}$ Out-Patient Department, Bangladesh Institute of Research and Rehabilitation in \\ Diabetes, Endocrine and Metabolic Disorders (BIRDEM), Dhaka, Bangladesh
}

\begin{abstract}
Background and objectives: The developing countries are facing the double burden of the communicable (CD) and non-communicable (NCD) diseases. The initiation of primary health care (PHC) adopted in the past century, which included sanitation and immunization, remarkably reduced the load of $\mathrm{CDs}$ in the least developing nations. The burden of NCDs remained the same or showed an increasing trend. Of the NCDs, diabetes has become a serious threat to human health and the related morbidity and mortalities are affecting the younger people. As a consequence, the disease complications render huge number of people to disabilities and unusual enormous health expenditures. Very few studies addressed the prevalence of complications among the diabetes patients in a rural community.

This study aimed to determine the prevalence of sequels (morbidity and mortality) among the diabetic cases eight years after the initial diagnosis of diabetes in a rural community cohort

Subjects and Methods: A rural community survey in 10 villages was conducted in 1993 . The survey screened 1319 (797 men, 522 women) for diabetes mellitus (DM) and impaired glucose tolerance (IGT). Those who were diagnosed DM and IGT referred to a referral center (BIRDEM) for registration. A retrospective cohort was designed in 2001. The addresses of the patients were retrieved from the BIRDEM registry. These registered patients, both survivors and nonsurvivors, were traced in ten villages. The survivors were investigated (anthropometry, glycemia, fundoscopy, urine protein etc.). A verbal autopsy was performed to determine the cause(s) of death in the non-survivors.

Results: Of the188 registered cases, 79 were found and located (survivors 43 (54.4\%, nonsurvivors 36 (45.6\%). Of the survivors, $44.2 \%$ developed complications. The observed complications were sensory neuropathy $16.3 \%$, CAD $9.3 \%$, retinopathy $7 \%$ and nephropathy 4.7\%. Among the non-survivors, $19.4 \%$ were found to have nephropathy leading to end-stage renal disease.

Conclusions: The study cohort revealed that more than one-third of the people with diabetes died in less than ten years after being diagnosed. The cohort also revealed that diabetic nephropathy (end-stage renal disease) and dearth of dialysis facilities contributed to early death in the rural community. Among the complications, most frequent incidence was neuropathy and neuro-psychiatric disorders.
\end{abstract}

IMC J Med Sci 2021; 15(1): 005

*Correspondence: M. Abu Sayeed, Department of Community Medicine, Ibrahim Medical College, 1/A Ibrahim Sarani, Segunbagicha, Dhaka-1000.email: sayeed@imc.ac.bd 


\section{Introduction}

In a recent report, World Health Organization (WHO) emphasized the alarming increasing trend of diabetes - rising from 180 million in 1980 to 422 million in 2014 [1]. During this time period, the prevalence of diabetes almost doubled from $4.7 \%$ to $8.5 \%$ in adult population [1-3]. The WHO also observed that premature death due to diabetes increased 5\% from 2000 to 2016 affecting mostly the low and middle income countries. A substantial number of studies observed various organ dysfunctions leading to morbidity and mortality of the diabetic patients. These findings were mostly based on the patients on regular follow-up either at the outpatient or the inpatient departments of the hospitals. Very few published reports are available regarding organ dysfunction or sequels among those who are diagnosed of having diabetes at population-based screening for diabetes in rural community. This study assessed the morbidity and mortality of diabetes in a rural community cohort eight years after the initial diagnosis.

\section{Materials and methods}

Ten villages of Kharua Union in Nandail sub-district under Mymensingh district, Bangladesh were surveyed in February and March 1993 to assess the prevalence of diabetes mellitus (DM). A total of 1319 subjects aged 18 years or more were screened for diabetes. Oral glucose tolerance test (OGTT) with $75 \mathrm{~g}$ glucose drink was used $[4,5]$ to diagnose diabetes. All newly detected DM and IGT subjects were referred to a referral center, Bangladesh Institute of Research and Rehabilitation in Diabetes, Endocrine and Metabolic Disorders (BIRDEM). The participants were registered and given a unique REFERENCE number. A second OGTT was done to confirm the diagnosis during registration. At registration, they were clinically examined, investigated and advised accordingly. Each participant was given special counseling for self-management of diabetes in a rural setting. They were encouraged to attend BIRDEM OPD, if necessary, and also for regular follow-up. An investigator maintained the communication with those registered patients.

After an interval of eight years in 2001, the REFERENCE numbers of those diabetic cases were retrieved from the BIRDEM registry. The addresses of the registered cases were collected and the cases were traced in the community. Some houses were missing or lost. The houses were missed or lost due to natural disaster like flood, river erosions, cyclone and migration to other areas. We interviewed the neighbors and the people of adjacent houses to locate his/her present migrant destination. Every effort was made to contact the registered diabetics, whether living or dead. For the dead individuals, we conducted a verbal autopsy using "2012 WHO verbal autopsy [form 3] death of a person aged 15 years and above" though some irrelevant questions were excluded or skipped. After locating them we investigated each individual for fasting plasma glucose, fundoscopy, urinary albumin, vibration and monofilament $(10 \mathrm{~g})$ tests and ECG to determine the presence of diabetes related sequels/complications. Any case, which had diabetes related complication(s) at the time of registration in 1993 were excluded from the follow up study of 2001 .

\section{Results}

A total of 1319 participants (797 men, 522 women) took part in the rural community survey in 1993. The methods and results were published [5]. The screening of participating population in 1993 detected 143 and 51 cases (total 194) of impaired glucose tolerance (IGT) and DM respectively using OGTT criteria of WHO. Of them, 188 subjects were registered in BIRDEM (Figure-1). We could locate only $79(42.0 \%)$ out of 188 cases. The survivors among them were $43(54.4 \%)$ and the nonsurvivors were 36 (45.6\%).

The biophysical characteristics of both men and women including comparisons between men and women are shown in Table-1. The men had significantly higher age $(p=0.009)$, height $(p<0.001)$ and serum creatinine $(p=0.033)$. In contrast, the women had higher BMI ( $p=0.003)$.

Table-2 depicted the possible causes of death in non-survivors and the clinical status of survivors of the diabetic cohort traced in 2001 after the initial diagnosis in 1993. Most deaths were reported to be due to diabetic nephropathy leading to end stage renal disease (19.4\%). The verbal communication 


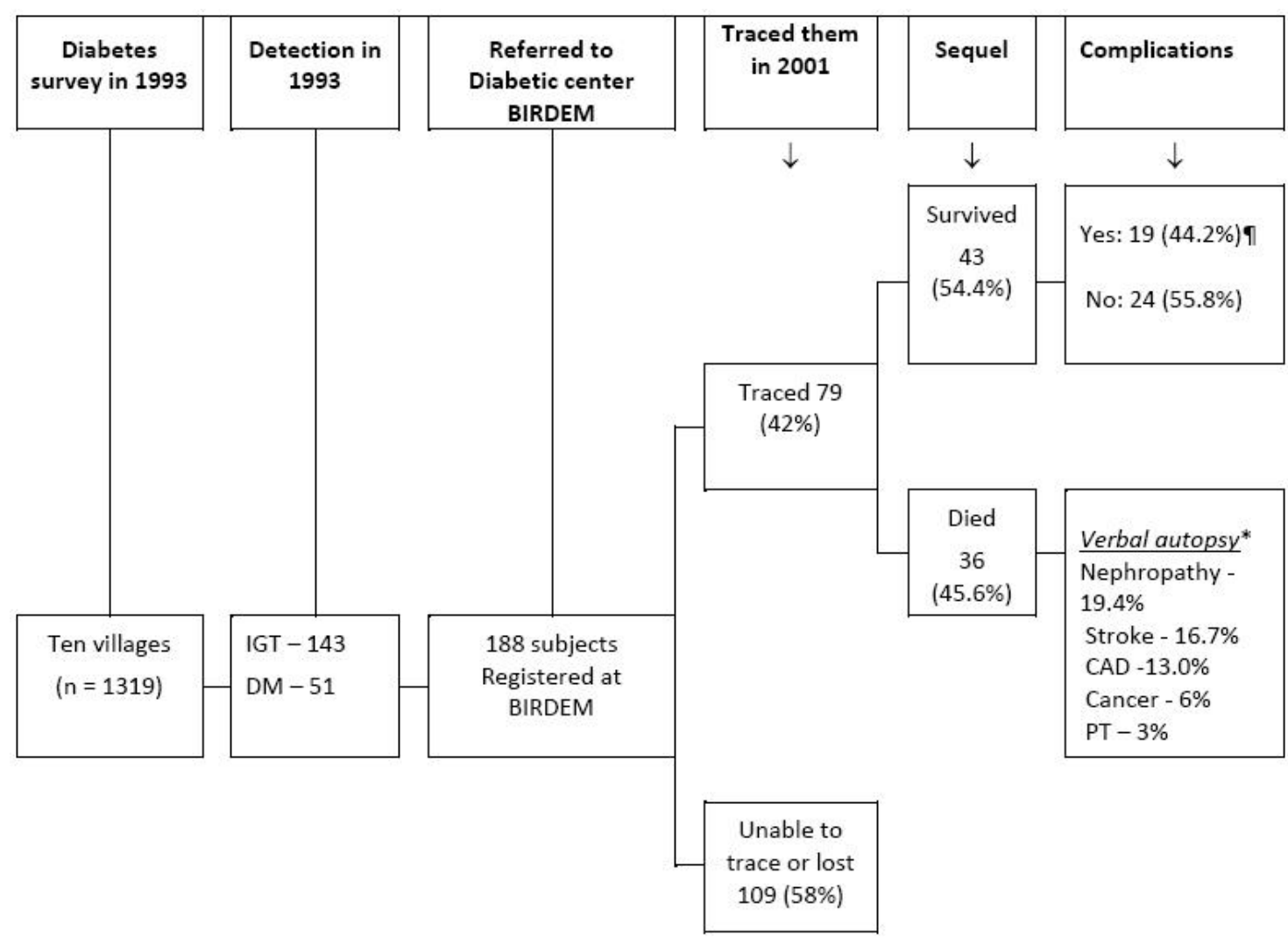

7-The complications depicted in Table-2; CAD - coronary artery disease; PT - Pulmonary tuberculosis

*Verbal autopsy also shown in Table-2.

Fig-1: Algorithm for participants who were first diagnosed as having impaired glucose tolerance or diabetes mellitus in 1993 and traced and investigated eight years later in 2001.

Table-1: Characteristics of the survivors $(n=43)$ and comparison between men and women (24/19)

\begin{tabular}{|c|c|c|c|c|c|c|c|}
\hline \multirow[t]{2}{*}{ Characteristics variables } & \multicolumn{2}{|c|}{$\begin{array}{c}\text { Both } \\
N=43\end{array}$} & \multicolumn{2}{|c|}{$\begin{array}{l}\text { Men } \\
N=24\end{array}$} & \multicolumn{2}{|c|}{$\begin{array}{c}\text { Women } \\
N=19\end{array}$} & \multirow[b]{2}{*}{$p^{\pi}$} \\
\hline & Mean & SD & Mean & SD & Mean & SD & \\
\hline Age (y) & 57.0 & 15.5 & 60.2 & 14.5 & 50.6 & 15.9 & .009 \\
\hline Height (cm) & 156.5 & 6.8 & 161.4 & 4.7 & 152.0 & 5.2 & .000 \\
\hline Weight (kg) & 47.0 & 7.9 & 44.1 & 5.1 & 49.7 & 9.2 & .088 \\
\hline Waist (cm) & 73.2 & 9.8 & 70.5 & 7.4 & 75.8 & 11.4 & .212 \\
\hline $\mathrm{Hip}(\mathrm{cm})$ & 82.2 & 5.1 & 82.0 & 4.5 & 82.5 & 5.8 & .812 \\
\hline Body mass index (BMI) & 19.3 & 3.9 & 16.9 & 2.3 & 21.5 & 3.99 & .003 \\
\hline Waist-to-hip ratio (WHR) & 0.88 & 0.08 & 0.85 & .07 & 0.91 & 0.09 & .130 \\
\hline Systolic blood pressure (mmHg) & 117.9 & 20.2 & 110.8 & 9.0 & 123.6 & 24.8 & .102 \\
\hline Diastolic blood pressure (mmHg) & 75.7 & 10.1 & 73.7 & 7.7 & 77.3 & 11.7 & .373 \\
\hline Fasting blood glucose (mmol/L) & 7.1 & 4.1 & 7.87 & 4.7 & 6.1 & 3.0 & .180 \\
\hline Blood glucose 2hPG (mmol/L) & 11.3 & 6.9 & 12.4 & 7.4 & 9.9 & 6.1 & 0.25 \\
\hline Serum creatinine $(\mathrm{mg} / \mathrm{dl})$ & 0.79 & 0.18 & 0.86 & 0.19 & 0.73 & 0.15 & .033 \\
\hline
\end{tabular}

" - $p$ after independent $t$ test: compared between men and women. 
Table-2: Causes of death in non-survivors and the clinical status of survivors of 79 registered diabetic cohorts traced in 2001after the initial diagnosis in 1993 (8 years interval)

\begin{tabular}{|c|c|c|}
\hline \multicolumn{3}{|l|}{ Non-survivors } \\
\hline Verbal autopsy based on history and death certificates & $\begin{array}{l}N=36 \\
(45.6 \%)\end{array}$ & $\%$ \\
\hline Generalized swelling (Nephropathy/renal failure) & 7 & 19.4 \\
\hline Died with hemiplegia (stroke) & 6 & 16.7 \\
\hline Sudden death (Coronary Artery Disease) & 5 & 13.0 \\
\hline Diagnosed cancer & 2 & 6.0 \\
\hline Jaundice +coma (hepatic coma) & 2 & 6.0 \\
\hline Congestive cardiac failure & 2 & 6.0 \\
\hline Pulmonary tuberculosis & 1 & 3.0 \\
\hline Nephropathy+retinopathy & 2 & 6.0 \\
\hline Death - cause of death could not be identified" & 7 & 19.4 \\
\hline Survivors with and without complications & $\begin{array}{l}N=43 \\
(54.4 \%)\end{array}$ & \\
\hline No complications (apparently healthy) & $24(55.8 \%)$ & \\
\hline Normal fasting glucose & & $03(6.9 \%)$ \\
\hline Impaired fasting glucose & & $05(11.6 \%)$ \\
\hline Diabetes mellitus & & $16(37.2 \%)$ \\
\hline Complications developed & $19(44.2 \%)$ & \\
\hline Sensory neuropathy & & $07(16.3 \%)$ \\
\hline Diabetic Retinopathy & & $03(7.0 \%)$ \\
\hline CAD (coronary artery disease, evidenced by ECG) & & $04(9.3 \%)$ \\
\hline Massive Proteinuria (Nephropathy) & & $02(4.7 \%)$ \\
\hline $\begin{array}{l}\text { No complication detected despite extreme } \\
\text { weakness (?psychiatric disorder) }\end{array}$ & & $03(7.0 \%)$ \\
\hline
\end{tabular}

indicated most of them were advised regular dialysis or kidney transplant. Neither regular dialysis nor kidney transplant was feasible or accessible to the families. The second most common cause was stroke $(16.7 \%)$. It was easy to clinch diagnosis as their deaths ensued after long sufferings with hemiplegia. Sudden death occurred in $13.0 \%$. These deaths appeared to be due to coronary artery disease (CAD) as described by the nearest relatives. Obesity, antihypertensive medication, smoking, sedentary habit and not complying the advices of treating physician were the main features of diagnosing CAD. Even after extensive interviewing and searching, cause(s) of death could not be identified in $19.4 \%$ of deaths. Of these cases, the nearest family members believed that the deaths were natural (extreme old age, bed-ridden for months or even years). One death was reported as paranormal (due to bad air). The apparently healthy person was found dead, while working in the nearest garden. One death occurred unattended when no relative or family member was present.

For the 43 survivors, $55.8 \%$ were found apparently healthy and no complications could be detected by physical examinations and investigations conducted at field levels. Striking observation was that almost $7 \%$ were found to have normal blood glucose (both fasting and post-prandial). Of the total survivors ( $n$ 43) almost half of them, remained as intermediate (pre-diabetes, $11.6 \%$ ) or DM (37.2\%). Of those who were found to develop complications (44.2\%), sensory neuropathy was most common (16.3\%) followed by CAD (9.3\%) and retinopathy (7.0\%). No 
organ dysfunctions or complications were detected in $3(7.0 \%)$. But these three diabetic individuals were found extremely weak, apathetic, anorexic and lost interest in life, totally withdrawn from family and social life. Possibly they had some neuro-psychiatric dysfunction developed as a consequence of metabolic disorder like hyperglycemia or dyslipidemia.

\section{Discussions}

This study may be considered as a unique one. The publications related to such a rural diabetic cohort are rare. Therefore, comparisons could not be made. However, some findings are noteworthy.

Firstly, the end-stage renal diseases leading to death was proved to be common among the diabetic population. Neither regular dialysis nor transplantation was affordable for the rural agrarian people. Thus, the findings emphasized the need of primordial or primary prevention, or at least an early diagnosis and intervention in order to avert such terminal events. It also underlines the necessity of availability and accessibility of dialysis facilities for the rural people.

Secondly, once diagnosed as diabetics or prediabetics, almost $50 \%$ of them remained such hyperglycemic for the rest of life or 8 years at least as the study suggested.

Thirdly, 7\% reversed to non-hyperglycemic. This invites query regarding the factors that influence reversal to non-diabetic state.

Finally, neurologic involvement was found to be the commonest among the survivors. If we take $16.3 \%$ of sensory neuropathy and other $7 \%$ as suspected neuropsychiatric disorder then it emphasizes the need for regular check up, neuropsychiatric examination and investigation.

The study has some important limitations. The characteristics were not compared between the initial findings recorded at BIRDEM registry (1993) and the last cohort findings (2001). Such comparisons of biophysical characteristics between two time points (1993 and 2001) could have provided some important factors contributing to the sequels (complications among survivors). Again, comprehensive examinations and investigations could reveal additional hematologic, hepatic, respiratory or neurological abnormalities.

\section{Conclusion}

Morbidity and mortality of diabetes related complications were high in the community. Early detection of diabetes by population-based screening and mere registration at a referral hospital was not adequate to prevent high mortality and morbidity. Of the mortality, diabetic nephropathy or end stage renal failure was the most common. This finding strongly suggests the need of regular follow up and care of diabetics including the accessibility of dialysis facilities for the rural people. Of the complications, neuropathy and neuro-psychiatric disorders proved to be important. It indicates the importance of neuropsychiatric care of the rural people.

\section{Acknowledgement}

We are indebted to the participants for volunteering this cohort. We recognize the contribution of the local people for helping us to locate and identify the diabetic patients screened in 1993 at the community level and registered at BIRDEM. Finally, we commemorate the two coauthors (Khandaker Abul Ahsan", Fazlul Haq ${ }^{4}$ ) for their outstanding contribution to make the study a success.

Fund: The study was funded by BIRDEM authority.

\section{Conflict of interest: None}

\section{Reference}

1. World Health Organization. Global report on diabetes. Geneva: World Health Organizations; 2016.

2. Wild S, Roglic G, Green A, Sicree R, King H. Global prevalence of diabetes: estimates for the year 2000 and projections for 2030. Diabetes Care. 2004; 27(5): 1047-1053. 
3. Divers J, Mayer-Davis EJ, Lawrence JM, Isom S, Dabelea D, Dolan L, et al. Trends in Incidence of Type 1 and Type 2 Diabetes among youths Selected Counties and Indian Reservations, United States, 2002-2015. MMWR Morb Mortal Wkly Rep. 2020; 69(6): 161-165.

4. Puavilai G, Chanprasertyotin S, Sriphrapradaeng A. Diagnostic criteria for diabetes mellitus and other categories of glucose intolerance: 1997 criteria by the Expert Committee on the
Diagnosis and Classification of Diabetes Mellitus (ADA), 1998 WHO consultation criteria, and 1985 WHO criteria. Diabetes Res Clin Pract. 1999; 44(1): 21-26.

5. Sayeed MA, Ali L, Hussain MZ, Rumi MA, Banu A, Azad Khan AK. Effect of socioeconomic risk factors on the difference in prevalence of diabetes between rural and urban populations in Bangladesh. Diabetes Care. 1997; 20(4): 551-555. 